\title{
Improved Magnetic Lateral Flow Assays with Optimized Nanotags for Point-Of-Use Inductive Biosensing
}

\author{
María Salvador ${ }^{1,2}$, Álvaro Gallo-Cordova ${ }^{3}$, Amanda Moyano ${ }^{1,4}, \mathrm{~J}$. \\ Carlos Martínez-García ${ }^{1}$, M. Carmen Blanco-López ${ }^{4}$, M. Puerto \\ Morales $^{3}$, Montserrat Rivas ${ }^{1}$ \\ ${ }^{1}$ Department of Physics \& IUTA, University of Oviedo, Campus de Viesques, 33204 Gijón, Spain \\ ${ }^{2}$ Istituto di Struttura della Materia - Consiglio Nazionale delle Ricerche (CNR), 00016 \\ Monterotondo Scalo (RM), Rome, Italy \\ ${ }^{3}$ Instituto de Ciencia de Materiales de Madrid (ICMM-CSIC), 28049 Madrid, Spain \\ ${ }^{4}$ Department of Analytical and Physical Chemistry, University of Oviedo, 33006 Oviedo, Spain
}

\begin{abstract}
Lateral flow assays may be used by minimally trained personnel for fast and inexpensive bioanalyses in decentralized non-exigent environments. Their extension to a broader catalog of applications depends on improvements in their quantification and their sensitivity. We report a strategy that combines nanomagnetic tagging of the analyte of interest with radiofrequency inductive sensing, easy to achieve in friendly and portable format. To optimize nanotag performance, we investigated the influences of their magnetic core size and agglomeration. Iron oxide nanoparticles, with sizes from 5 to 23 $\mathrm{nm}$, were synthesized by thermal decomposition and then coated with dimercaptosuccinic acid and functionalized with neutravidin protein. We tested the system by immobilizing biotin in lateral flow membrane strips. When a sample containing the particles flows along the membrane, the biotin captures the neutravidin together with the magnetic nanotags, which are detected by the inductive sensor. The optimal nanotag core size is the critical threshold for superparamagnetic behavior, which maximizes both the initial magnetic permeability and the saturation magnetization. Controlled agglomeration of the nanotags increases the magnetic mass captured in the test line and further amplifies the signal.
\end{abstract}

\section{Introduction}

Lateral flow immunoassay is a bio-testing method that is spreading thanks to its many advantages for point-of-care applications, such as quickness, portability, easy use, and low cost. Since its well-known use for home pregnancy tests launched in the early 1980s, its increasing application for diagnosis and prognosis in health ${ }^{1-4}$ and food and environmental safety ${ }^{5-9}$ have conferred it a solid reputation as a routine screening tool. In applications that need rapid decision-making ${ }^{10-12}$ the advantages of the lateral flow method stand up, even when sometimes its sensitivity is lower than that of other immunoanalytical techniques (e.g., the enzyme-linked immunosorbent assay). To improve sensitivity and reduce the limits of detection, some ideas, such as chemical signal enhancement, ${ }^{13-15}$ test design, ${ }^{16}$ or more sensitive transducers or read-out instrumentatio ${ }^{17}$ have been explored. 
The keys of lateral flow assays (LFA) are paper microfluidics and bio-recognition. The test consists of a strip of a nitrocellulose nanoporous membrane along which the liquid sample (urine, saliva, blood, serum, or plasma, or food or environmental samples) flows by capillary action. The analyte of interest is selectively captured by a bio-recognition molecule that is previously immobilized across the strip, forming the test line. In order to develop the test, the bioreceptor used for detection is labeled by colored nanoparticles that are detectable by the naked eye and provide a yes/no response. The presence/absence test or a semiquantitative one is satisfactory for some applications such as pregnancy, but for many others, such as diagnosis by biomarkers in cancer or myocardial infarction and toxin thresholds, one needs reliable, quantitative results.

Optical readers based on image analysis, reflectance or fluorescence measurements can be used to quantify the signal. ${ }^{18,}{ }^{19}$ However, these readings are very sensitive to ambient light, humidity, and staining or aging of the paper strip, which frequently cause difficulties in calibration and reproducibility, especially in samples with a complex or strongly colored matrix. ${ }^{20}$

Some authors have proposed magnetic nanoparticles (MNPs) as an efficient label in $\mathrm{LFA}^{18}$. MNPs have a tunable size and surface chemistry, which make them physically and chemically stable, biocompatible and easily biofunctionalizable. Additionally, their production is not expensive. ${ }^{21}$ MNPs produce a magnetic perturbation around them that can be detected by an adequate magnetic sensor without interference from the biological sample or the paper. Moreover, one can use their magnetism for pre-concentration or separation of the target analyte from the sample matrix, which can enormously help enhance the sensitivity without complex manipulation. ${ }^{22-24}$ Additionally, magnetic signals do not degrade significantly with time, and sense not only particles on the surface but in the whole volume of the test line. ${ }^{25}$

We recently developed a detection method using superparamagnetic nanoparticles combined with inductive detection with a single planar coil. ${ }^{26}$ We proved the feasibility of the methodology in the quantification of prostatic cancer biomarkers ${ }^{27}$ and toxic biogenic amines. ${ }^{20}$

To optimize magnetic LFA, we need to analyze the properties of the separate components as well as their cooperative behavior. In inductive detection, the most critical parameters of the nanoparticles should be their magnetic moment and their initial magnetic permeability at the working frequency. To determine the possibility to control these variables, we have studied in this work the influence of the magnetic core size of the nanoparticles on their efficiency as LFA labels. For this purpose, iron oxide nanoparticles with sizes ranging from 5 to $23 \mathrm{~nm}$ were synthesized, characterized, and calibrated in the magnetic sensor. We have then tested them in lateral flow assays by using the model system neutravidin-biotin. We want to remark that neutravidin-biotin affinity has long be used in lateral flow immunoassays for the detection of clinical analytes as many commercial antibodies are biotinylated. Therefore, here it is used as a dummy in which the target of the detection is the neutravidin.

\section{Materials and methods}




\subsection{Chemicals and Reagents}

Iron chloride hexahydrate. oleic acid, sodium oleate, ethanol, toluene, hexane, octadecene, dimethyl sulfoxide (DMSO), meso-2,3-dimercaptosuccinic acid (DMSA), 1ethyl-3-[3-di-methylpropyl]carbodiimide (EDC), bovine serum albumin (BSA), biotinconjugated bovine serum albumin (BBSA) and Tween20 were purchased from SigmaAldrich (Spain). Neutravidin protein was obtained from Thermo Fischer Scientific (USA).

\subsection{Magnetic Nanoparticles Synthesis and Characterization}

The synthesis of MNPs with different core sizes was carried out by thermal decomposition using iron oleate as an iron precursor, which was prepared by a modification of the procedure published by Bronstein et al. ${ }^{28}$ In a typical experiment, 10.8 g of $\mathrm{FeCl}_{3} \cdot 6 \mathrm{H}_{2} \mathrm{O}$ were mixed with $45 \mathrm{~g}$ of sodium oleate in $60 \mathrm{~mL}$ of distilled water, 80 $\mathrm{mL}$ of ethanol, and $140 \mathrm{~mL}$ of hexane. The mixture was heated to $343 \mathrm{~K}$ and the reaction was left for 4 hours in a well-sealed system. Once the mixture was cooled, the aqueous phase was separated and discarded with a separation funnel and the final product was washed 3 times with distilled water. The remnant hexane and ethanol were evaporated by using a rotary evaporator. The final product was left in an inox-oven at $323 \mathrm{~K}$ for $12 \mathrm{~h}$.

For MNPs preparation, $4.5 \mathrm{~g}$ of liquid iron oleate were weighted with $1.4 \mathrm{~g}$ of oleic acid and mixed in $50 \mathrm{~mL}$ of octadecene. The mixture was then placed in a three-neck roundbottom flask in nitrogen environment. First, the mixture was agitated at $340 \mathrm{rpm}$ and heated to $333 \mathrm{~K}$ so the reactants could dissolve. Then, the mixture was heated at a rate of $3.4 \mathrm{~K} / \mathrm{min}$ until the octadecene boiling point was reached $(593 \mathrm{~K})$, where the reaction was left for one hour. The sample was collected by centrifugation at $8000 \mathrm{rpm}$ for $15 \mathrm{~min}$ and then washed several times with ethanol until organic precursors and reactants were removed. The obtained MNPs were dispersed in toluene for further functionalization. The final particle size was increased by reducing the amount of oleic acid in the reaction media. ${ }^{29}$ Specifically, $1.4,0.7$, and $0 \mathrm{~g}$ of oleic acid were used to achieve particle sizes around 8,12 , and $23 \mathrm{~nm}$ respectively.

The MNPs were coated by DMSA by a ligand exchange process to remove the oleic acid. ${ }^{30}$ A previously prepared mixture of $90 \mathrm{mg}$ of DMSA with $5 \mathrm{~mL}$ of DMSO was added into $20 \mathrm{~mL}$ of a MNP dispersion of $2.5 \mathrm{mg} / \mathrm{mL}$. After 24 hours of mechanical stirring, the solvent was discarded and the precipitated MNPs were collected and washed three times with ethanol. Afterwards, the $\mathrm{pH}$ of the MNPs dispersion was increased to 10 with a 0.25 $\mathrm{M} \mathrm{NaOH}$ solution and dialyzed and filtered through a $0.22 \mu \mathrm{m}$ pore-size filter before adjusting the $\mathrm{pH}$ to 7 . The iron concentration was measured by inductively coupled plasma optical emission spectroscopy (ICP-OES) with an apparatus from Perkin Elmer, model OPTIME 2100DV, after digestion with aqua regia. The surface chemistry and nature of the iron oxide nanoparticles were studied using a Nicolet FT-IR 20SXC spectrometer recorded in the range of $400-4000 \mathrm{~cm}^{-1}$. Powdered samples were mixed with $\mathrm{KBr}$ and pressed in pellets.

Colloidal properties of the MNPs were studied in a Malvern Instruments Zetasizer Nano SZ by dynamic light scattering (DLS) measurement equipped with a solid-state $\mathrm{He}-\mathrm{Ne}$ laser (wavelength $\lambda=633 \mathrm{~nm}$ ) that provided the hydrodynamic size distribution and average $\zeta$-potential. The magnetic core size was obtained by transmission electron 
microscopy (TEM) using a JEOL JEM 1010 microscope at $100 \mathrm{keV}$. For sample preparation, a drop of a dilute particle suspension was placed on a copper grid coated with amorphous carbon and then the solvent was left for evaporation at room temperature. The TEM particle size distributions were evaluated by measuring the largest core dimension of at least 200 particles. The data were fitted to a lognormal distribution from which the mean size and the standard deviation were obtained.

The magnetic properties of the MNPs were studied using a vibrating sample magnetometer MagLabVSM, Oxford Instruments, with a maximum field of $5 \mathrm{~T}$. A known amount of a sample was dried at $323 \mathrm{~K}$ for $12 \mathrm{~h}$, and then placed in the sample holder. The hysteresis loop of the samples was measured at $290 \mathrm{~K}$ up to $\pm 5 \mathrm{~T}$. Following the same sample protocol preparation at room temperature, zero-field-cooled (ZFC) and field-cooled (FC) curves were obtained using a Quantum Design PPMS magnetometer equipped with a superconducting coil that produces magnetic fields in the range from -14 $\mathrm{T}$ to $+14 \mathrm{~T}$. Initial magnetic susceptibility was measured with the same device from 1 $10,000 \mathrm{~Hz}$. The magnetization values given in this work are referred to the mass of $\mathrm{Fe}_{3} \mathrm{O}_{4}$ derived from the iron concentration obtained by ICP-OES analys is.

To evaluate the MNPs in the LFA's scanning inductive sensor, some droplets of known mass from each sample were deposited onto a $10 \mathrm{~mm} \times 2 \mathrm{~mm}$ blotting paper and left to dry for at least $12 \mathrm{~h}$.

\subsection{Nanoparticle Biofunctionalization}

MNPs of three representative core sizes $(8,12$, and $23 \mathrm{~nm})$ and different degrees of agglomeration were functionalized with neutravidin and tested on an LFA across which we had printed a biotin test line.

The neutravidin-biotin system is here used as a model to assess the performance of the particles as tags. The biotin-neutravidin combination is widely known in biochemistry for its high affinity constant, high thermal and chemical stability, and low non-specific binding. Neutravidin-conjugated magnetic labels are attractive because many biotinylated antibodies are commercialized for immunoassays. ${ }^{31,} 32$ They are also used as a signal amplification method. ${ }^{15,33}$

Here, we use this binding as the antibody-antigen paradigm of the immunoassay. The carboxylic group present in the DMSA that covers the MNPs was used to link the neutravidin via an EDC-mediated coupling reaction. We adapted the protocol from. ${ }^{34}$ Briefly, $1 \mathrm{mg}$ of EDC was dissolved in $1 \mathrm{~mL}$ of phosphate-buffered saline (PBS, $1 \mathrm{mM}$ $\mathrm{pH}$ 7.4) freshly prepared and kept refrigerated during the process. A solution of neutravidin $(1 \mathrm{mg} / \mathrm{mL})$ was prepared and mixed with a certain amount of the MNPs. After being placed in a refrigerated ultrasonic bath, subsequent $10 \mu \mathrm{L}$ additions of the EDC solution were done at $0,2,4,6,8,24$, and 30 hours. We carried out DLS measurements to monitor the biofunctionalization of the particles with neutravidin.

\subsection{Preparation of Lateral Flow Strips}

For the LFA assembly, we purchased nitrocellulose membranes (UniSartCN95, Sartorius, Spain), glass fiber sample pads (GFCP001000, Millipore, Germany), absorbent pads (Whatman, USA) and backing cards (KN-V1080, Kenoshatapes, Netherlands). 
The nitrocellulose membrane ( $25 \mathrm{~mm}$-wide) was attached to an adhesive backing plastic card to make it sturdy. To form the test line at which the neutravidin will be immobilized (together with the magnetic labels), we have dispensed across the nitrocellulose membrane a solution of $1 \mathrm{mg} / \mathrm{mL}$ of BBSA at a rate of $0.100 \mu \mathrm{L} / \mathrm{mm}$ (with an IsoFlow reagent dispensing instrument, Imagene Technology, USA.) After drying, the sample pad (which enables a controlled transfer of the sample to the membrane) and the absorbent pad (which acts as a wick and prevents the backflow) were placed onto the backing card with an overlap of $2 \mathrm{~mm}$. Single $5 \mathrm{~mm}$ wide strips were cut with a guillo tine (Fellowes Gamma, Spain).

To obtain the sample solution, $80 \mu \mathrm{L}$ of freshly prepared running buffer (RB) containing $10 \mathrm{mg} / \mathrm{mL}$ BSA and $0.5 \%$ Tween20 in PBS $(10 \mathrm{mM}, \mathrm{pH} 7.4)$ were mixed with $20 \mu \mathrm{L}$ of the neutravidin-MNP conjugate. The tests were then carried out in dipstick format by vertically introducing the sample pad end in the sample solution. The solution flows up by capillary action, and the neutravidin gets trapped by the biotin in the test line (see Figure 1). After $10 \mathrm{~min}$ of immersion, the strip was taken out and let dry.

A)

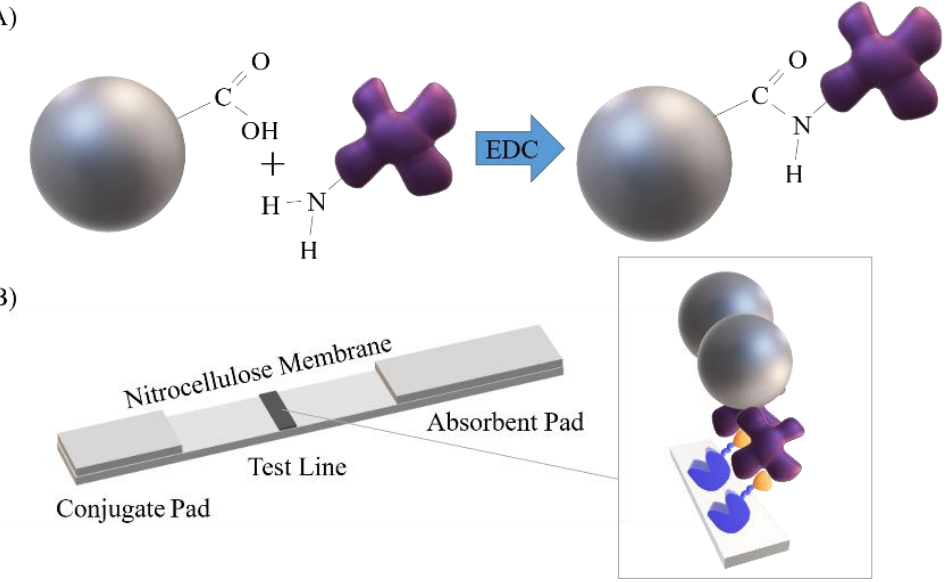

Figure 1. A) Schematic illustration of the MNPs biofunctionalization with neutravidin by EDC chemistry. B) Scheme of a lateral flow strip for neutravidin capture via biotin affinity. For simplification, only one$\mathrm{COOH}$ group has been drawn at the MNPs, and only one $-\mathrm{NH}_{2}$ group at the neutravidin.

\subsection{Quantification of the Immunoassays}

We evaluated the magnetic LFA signal by means of an inductive sensor that was developed specifically for lateral flow strips (see Figure 2) ${ }^{27}$ Its sensing head consists of a double copper line printed on a rigid insulating substrate across which an alternating current flows. The magnitude and phase of the sensing head impedance are continuously monitored by a precision impedance analyzer (Agilent 4294A) using $16048 \mathrm{G}$ test leads and $500 \mathrm{mV}, 20-110 \mathrm{MHz}$ excitation voltage. 
A)

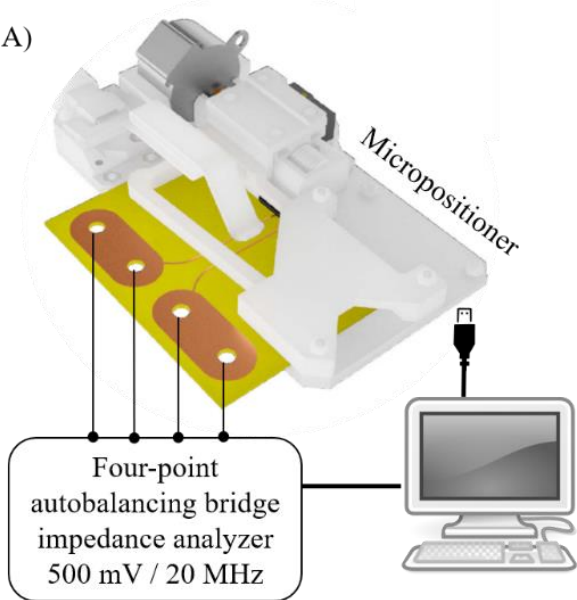

B)

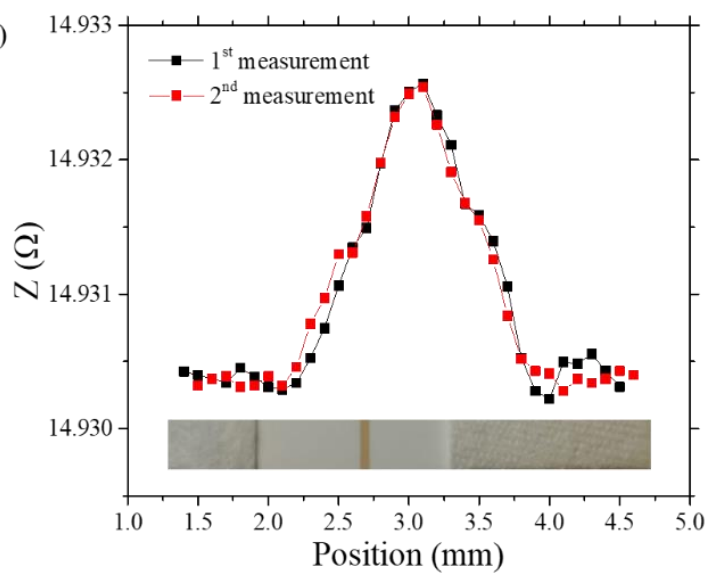

Figure 2. A) Schematic representation of the inductive sensor. B) Sensor signal for two scannings of the test line of an LFA. Bottom: image of the LFA where the brownish test line can be seen.

The sensing planar coil can be approximated by an RL circuit whose electrical impedance depends on the frequency $v$ and magnetic relative permeability $\mu_{r}$ of the surrounding medium, as:

$$
Z(v, \mu)=R(v)+i 2 \pi v L \mu_{r}(v)
$$

where the resistive part $R(v)$ depends on the frequency due to the skin effect, and $L$ is the self-inductance, dependent on the geometry of the conductor and any surrounding magnetic material. In absence of any magnetic particles, the magnetic permeability can be approximated by the vacuum permeability, $\mu_{r}=1$. When the sensing coil is completely covered by a magnetic material with an initial susceptibility $\chi(v)$, the relative permeability becomes $\mu_{r}(v)=\chi(v)+1$. In the present application, the magnetic particles do not surround the whole length of the conductor, hence, a correction factor $\psi(\psi<1)$ must be included to account for the volume of particles. Taking this into account, the difference in impedance measured with and without the particles can be written as:

$$
\Delta Z\left(v, \chi^{\prime}, \chi^{\prime \prime}, \psi\right)=v L \psi \chi^{\prime \prime}(v)+i v L \psi \chi^{\prime}(v)
$$

where $\chi^{\prime}$ and $\chi^{\prime \prime}$ stand for the real and imaginary components of the magnetic initial susceptibility of the magnetic material (we want to highlight that due to the product of the two complex numbers, $Z$ and $\chi$, the real part of $\Delta Z$ depends on the imaginary component of the susceptibility and vice versa.) According to this, for a fixed frequency, both the real and the imaginary parts of the impedance variation are directly proportional to the mass of the magnetic material through $\psi$. This linear dependence has been checked in previous works. ${ }^{26}$

The test lines of the LFAs were scanned laterally over the sensing coil with a micropositioner, producing a peak in impedance whose width is related to the width of the line. We integrate the peak signal across the position to account for all the particles, 
disregarding their distribution in the test line (with this we avoid inaccuracies coming from dispensing flaws.) The signal $S$ provided by the sensor is then obtained in units of $\Omega \cdot \mathrm{mm}$ coming from the cumulative integral of the impedance $(\Omega)$ across the width $w$ of the test line $(\mathrm{mm})^{26,27}$ (see Figure 2B):

$$
S=\int_{0}^{w} \Delta Z d x
$$

We define the resolution $R$ of the method as the smallest change in mass that our sensor can resolve:

$$
R=\frac{m \sigma_{\text {noise }}}{\Delta Z}
$$

where $\Delta Z=Z-Z_{0}$ is the variation of the impedance with and without MNP on the sensor. The sensitivity $\Sigma$ can be calculated following the use of giant magnetoimpedance and magnetoresistance sensors like:

$$
\Sigma=\frac{1}{m} \frac{\Delta Z}{Z_{0}} 100
$$

\section{Results and discussion}

Crossing, equations (4) and (5) with (2), we can see that both $\Sigma$ and $R$ depend on the characteristics of the MNP that are used to label the biomolecule in the test strip (in this paper, the neutravidin.) The two variables that affect the sensitivity of the method are (i) the initial magnetic susceptibility $\chi$ and (ii) the total volume of the MNPs that we can attach to each molecule (directly proportional to $\psi$.) While the latter depends on the bioconjugation and immobilization of at the LFA, the former is an intrinsic property of the particles. For this reason, we have done a thorough investigation of the optimal characteristics for inductive detection of the unmodified MNP.

We evaluated nine sample solutions with different average particle sizes ranging from 5 to $23 \mathrm{~nm}$ with the inductive sensor. The dependence of $\Sigma$ and $R$ with the initial susceptibility $\chi(v=10 \mathrm{~Hz})$ is plotted in Figure 3 . These results have been obtained at 20 $\mathrm{MHz}$ (although the sensitivity increases with frequency, also does the level of noise, which considerably worsens the resolution.) The linear correlation between $\Sigma$ and $\chi$ applies to all the particles except one: The values for $23 \mathrm{~nm}$ do not follow the general trend, and both $\Sigma$ and $R$ are worse for this sample.

A likely explanation for the worse performance of the $23 \mathrm{~nm}$ particles is an excessive particle size. Particles with sizes below $50 \mathrm{~nm}$ are magnetic monodomains, whose magnetic moment lies along the easy magnetization direction determined by the crystallographic structure and the shape. Switching the magnetic moment from one direction to the opposite requires an energy that depends on the nature of the particle through its magnetic anisotropy constant $K$ and volume $V: E=K V$. When $V$ is very small, it is probable that the thermal energy $k_{B} T$ (where $k_{B}$ stands for Boltzmann constant and $T$ for the temperature) enables the switching at such a high rate that, for many measurements and practical applications, the observed magnetization vanishes. This behavior is known as superparamagnetism $\mathrm{m}^{35}$ and occurs above a critical volume (for 
a given temperature), or a transition temperature known as blocking temperature $T_{B}$ (for a given volume). Particles whose combination of volume and temperature leaves them out of the superparamagnetic regime are said to be blocked. Superparamagnetism significantly affects the susceptibility of the particles. ${ }^{36}$ A substantial magnetic susceptibility is essential for inductive detection. On the other hand, the operation frequency must be high (10-200 MHz) to get a measurable signal. At such frequencies it is more difficult for the magnetization to follow the exciting field than at $10 \mathrm{~Hz}$. This lag provokes the reduction of the susceptibility and the appearance of an out-of-phase component (mathematically described by $\chi^{\prime \prime}$.) Thanks to the thermal excitation, the magnetic susceptibility of superparamagnetic particles at high frequencies remains larger than that of their ferri- or ferromagnetic counterparts. Then, we presume that the sample with average size of $23 \mathrm{~nm}$ contains a large proportion of particles that are blocked and worsen the inductive sensor signal. On the contrary, particles of 12-14 nm optimize the signal. To confirm this, we have studied the magnetic behavior and the initial susceptibility of the particles at high frequency.

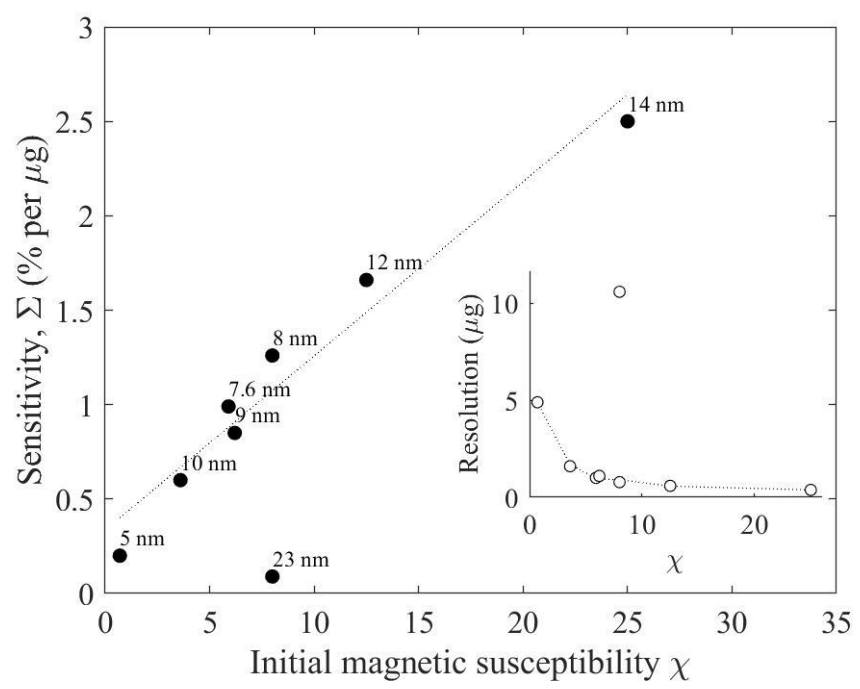

Figure 3. Sensitivity $\Sigma$ of the sensor at $20 \mathrm{MHz}$ as a function of the initial magnetic susceptibility $\chi(10 \mathrm{~Hz})$ of the nanoparticles. Inset: Resolution versus $\chi$. In both graphs, the point that goes out of the general trend corresponds to the $23 \mathrm{~nm}$ particles, which are not superparamagnetic.

\subsection{Magnetometry}

From here on, the discussion will focus on a selection of three particle samples, with average sizes of 8,12 , and $23 \mathrm{~nm}$, named NP8, NP12, and NP23, respectively. The nature of these nanoparticles is mainly magnetite with different degrees of oxidation as a function of the particle size. The oxidation is a consequence of the transference of the particles to water by ligand exchange with DMSA, followed by infrared spectroscopy. Thus, IR spectrum confirms the presence of DMSA on the surface and shows a certain degree of oxidation, more critical for particles smaller than $10 \mathrm{~nm}$ (NP8) (see Supplementary Information S2.)

Figure 4 shows their magnetization curves at room temperature. For NP8 and NP12, they are anhysteretic, consistent with superparamagnetic behavior, while NP23 has magnetic hysteresis (the inset in Figure 4 shows clearly the opening of the magnetization curve of NP23.) The saturation magnetization $M_{\mathrm{S}}$ was calculated by fitting the 
experimental data of Figure 4 to the law of approach to saturation. ${ }^{37}$ The results for the three types of particles, given in Table 1 , are close to that of bulk magnetite $\left(M_{\mathrm{S}}=98\right.$ $\left.\mathrm{A} \cdot \mathrm{m}^{2} / \mathrm{kg}\right) \cdot{ }^{38}$

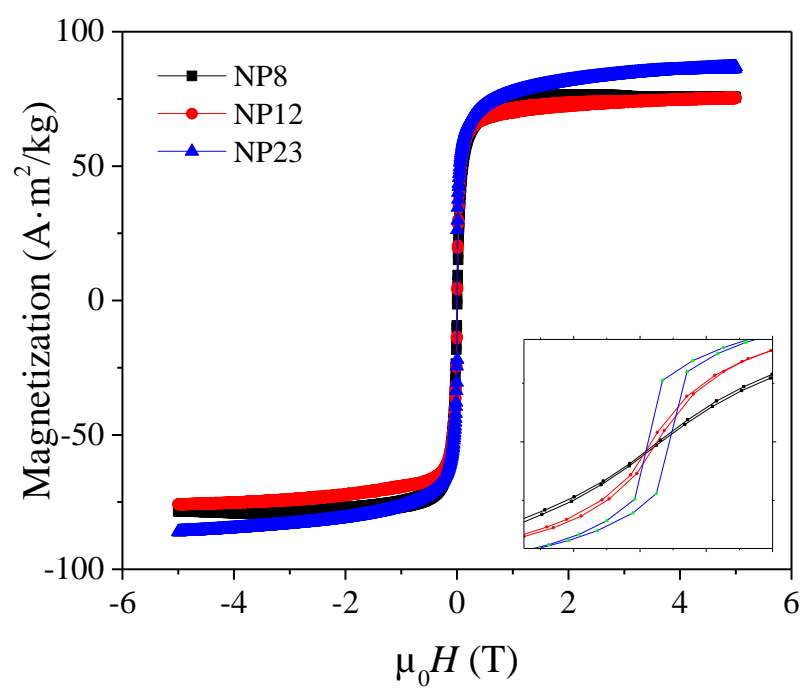

Figure 4. Hysteresis loops of the three samples NP8 (black), NP12 (red) and NP23 (blue) at room temperature from 5 to $-5 \mathrm{~T}$. Inset: Central area detail of the magnetization curves.

To assess the superparamagnetic behavior of the particles, we recorded the ZFC-FC curves, shown in Figure 5A, in an applied magnetic field of 5 mT. For NP8 and NP12, the ZFC curve shows a maximum below $200 \mathrm{~K}$, confirming that, at room temperature, in these solutions, most particles are in the superparamagnetic regime. On the contrary, for NP23, the slope of the ZFC curve is positive even at $350 \mathrm{~K}$. We obtained the blocking temperature $T_{\mathrm{B}}$ at which the particles transit from blocked to the superparamagnetic regime from the distribution of $T_{B}$ obtained as the temperature derivative of the differe nce $M_{\mathrm{ZFC}}-M_{\mathrm{FC}}$ of both curves $^{39}$ (see Figure $5 \mathrm{~B}$.) $T_{B}$ was calculated as the peak of the normal-curve fit. For monodisperse populations with homogeneous magnetic anisotropy, the two curves should coincide above $T_{\mathrm{B}}$; their separation, quantified by the difference between the temperature of the maximum $T_{\max }$ and the temperature at which both curves merge $T_{\text {irr }}$, is related to the width of the blocking temperature distribution, and hence, a measure of the particle size distribution. ${ }^{40}$ The effective anisotropy constant $\left(K_{\text {eff }}\right)$ can be then estimated as $K_{\text {eff }}=25 k_{\mathrm{B}} T_{\mathrm{B}} / V k_{\mathrm{B}}$ being the Bolzman constand $V$ the average volume of the particles-. ${ }^{41}$ The values obtained, shown in Table 1, are on the order of magnitude of published data for magnetite nanoparticles. ${ }^{42}$ The largest anisotropy corresponds to the smallest particles with an extra anisotropy source coming from the surface and the smallest value for the largest particles and very close to the magnetocrystalline anisotropy for bulk magnetite $\left(1.1 \times 10^{4} \mathrm{~J} / \mathrm{m}^{3}\right)$. 

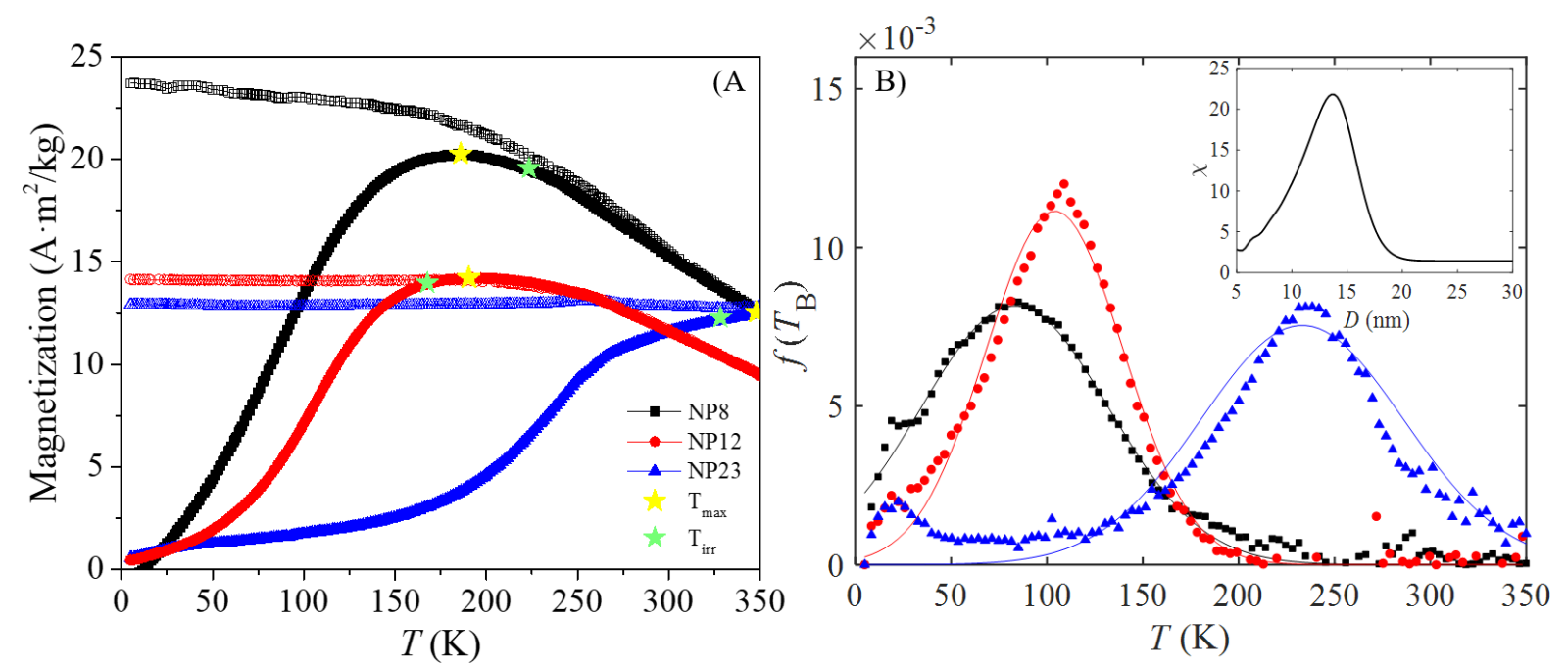

Figure 5. A) ZFC-FC curves for particles NP8 (black), NP12 (red) and NP23 (blue), obtained under a magnetic field of $5 \mathrm{mT}$. $T_{\max }$ (yellow star) and $T_{\text {irr }}$ (green star) are indicated for the three samples; B) Distribution of blocking temperatures; Inset: Calculated magnetic susceptibility as a function of the size. The legend is common to both graphs.

\subsection{Initial Magnetic Susceptibility}

To estimate the size that would maximize the initial magnetic susceptibility, we may use Néel's model. The susceptibility of an ensemble of MNPs with randomly oriented easy axes can be calculated at temperature $T$ according to equation (2) in ref. ${ }^{36}$ for null frequency,

$$
\chi(T)=\int_{0}^{\infty} \frac{2}{3} \frac{\mu_{0} \rho^{2} M_{S}^{2}}{K_{\mathrm{eff}}}\left(\frac{K_{\mathrm{eff}} V}{k_{B} T}+1\right) \mathfrak{L}\left(D, D_{0}, \sigma\right) d D
$$

in which we have included the log-normal distribution function $\mathfrak{L}\left(D, D_{0}, \sigma\right)$ to account for the polydispersion of the particles size $D . D_{0}$ is the mean size, and $\sigma$ is the standard deviation of the logarithm (see size analysis in the supplementary information file). We have used the parameters typical of magnetite particles (density $\rho=5170 \mathrm{~kg} / \mathrm{m}^{3}, K_{\text {eff }}=$ $5 \times 10^{4} \mathrm{~J} / \mathrm{m}^{3}$ and $\left.M_{S}=80 \mathrm{~A} \cdot \mathrm{m}^{2} / \mathrm{kg}\right), \sigma=0.01$, and $T=300 \mathrm{~K}$. The curve, plotted in the inset of Figure $5 \mathrm{~B}$, presents a maximum at $D_{0}=12 \mathrm{~nm}$. The AC susceptibility measurements confirm this prediction, as shown in Table 1.

Table 1. Saturation magnetization $M_{\mathrm{S}}$, blocking temperature $T_{\mathrm{B}}$, maximum temperature $T_{\max }$, effective anisotropy constant $K_{\mathrm{eff}}$, and real and imaginary components of the initial susceptibility (dimensionless), $\chi^{\prime}$ and $\chi^{\prime \prime}$, respectively.

\begin{tabular}{|c|c|c|c|c|c|c|c|}
\hline $\begin{array}{c}\text { Particle } \\
\text { Sample }\end{array}$ & $\begin{array}{c}\boldsymbol{M}_{\boldsymbol{S}} \\
\left(\mathbf{A} \cdot \mathbf{m}^{2} / \mathbf{k g ~ F e}_{3} \mathbf{O}_{4}\right)\end{array}$ & $\begin{array}{c}\boldsymbol{T}_{\boldsymbol{B}} \\
(\mathbf{K})\end{array}$ & $\begin{array}{c}\boldsymbol{T}_{\max } \\
(\mathbf{K})\end{array}$ & $\begin{array}{c}\boldsymbol{T}_{\text {irr }} \\
(\mathbf{K})\end{array}$ & $\begin{array}{c}\boldsymbol{K}_{\text {eff }} \\
\left(\mathbf{J} / \mathbf{m}^{3}\right)\end{array}$ & $\chi^{\prime}$ & $\chi^{\prime \prime}$ \\
\hline NP8 & 79 & 87.1 & 182.6 & 128.6 & $8.8 \cdot 10^{4}$ & 12.85 & 0.15 \\
\hline NP12 & 75 & 101.3 & 186.8 & 178.5 & $3.8 \cdot 10^{4}$ & 18.48 & 0.01 \\
\hline NP23 & 83 & 224.9 & $>350$ & 342.8 & $1.2 \cdot 10^{4}$ & 8.03 & 0.87 \\
\hline
\end{tabular}


Due to the inductive character of the magnetic sensor, the signal grows with the excitation frequency $(\Delta Z \propto v$, see equation (2)). Then, higher frequencies would, in principle, benefit the detection of the MNPs. This idea makes it interesting to analyze the behavior of the susceptibility in the sensor working frequency range $(10-200 \mathrm{MHz})$. To account for the influence of the frequency $v$, equation (6) can be modified as follows:

$$
\chi(\nu, T)=\int_{0}^{\infty} \frac{2}{3} \frac{\mu_{0} \rho^{2} M_{S}^{2}}{K_{\mathrm{eff}}}\left(\frac{K_{\mathrm{eff}} V /\left(k_{B} T\right)}{1+i 2 \pi v \exp \left(K_{\mathrm{eff}} V / k_{B} T\right)}+1\right) \mathfrak{L}\left(D, D_{0}, \sigma\right) d D
$$

The solution of equation (7) was numerically computed for the three types of particles by a trapezoidal method, using the size parameters given from the TEM histograms (see supplementary information $\mathrm{S} 1$ ), and $M_{\mathrm{S}}$ and $K_{\mathrm{eff}}$ from DC magnetization measurements (Table 1). The results are shown in Figure 6, where the dashed vertical lines delimit the working frequency range of the inductive sensor. In this scope, NP12 presents the highest and NP23 the lowest susceptibility values.

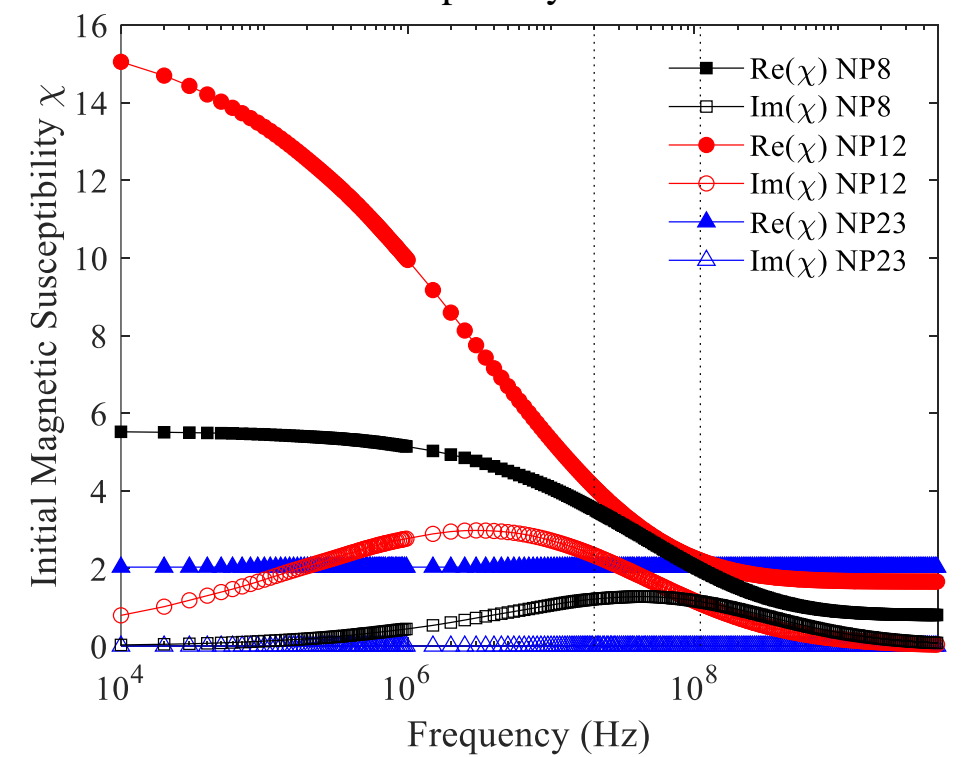

Figure 6. Computed frequency evolution of the initial susceptibility for samples NP8 (black), NP12 (red) and NP23 (blue).

These calculations can be used to explain the sensor signal from the various particles. As we can see from equations (2) and (3), the signal $S$ is proportional to the susceptibility with a multiplying factor that includes the MNPs mass (through $\psi$ ) and $v$, so plotting $S /(m v)$ (Figure 7) versus $v$ should give the same type of curves as the simulations of Figure 6 (we must recall that the real part of $S$ is proportional to $\chi^{\prime}$ and the real part of $S$ to $\chi^{\prime \prime}$.) We can observe effectively the similarities of the curves in the range of common frequencies $(20-110 \mathrm{MHz})$ : (i) The real part of the signal, which is related to $\chi^{\prime \prime}$, is much smaller than the imaginary component and barely changes (oscillations are attributable to noise;) (ii) The imaginary part of the signal decreases with the frequency, NP12 showing the largest and NP23 the smallest values. This allows us to confirm that, on equal terms of composition, mass, and coating, the initial susceptibility is a decisive parameter for detection. Given that the maximum susceptibility is achieved for the critical volume for 
superparamagnetism, this seems to be also optimal for inductive detection.
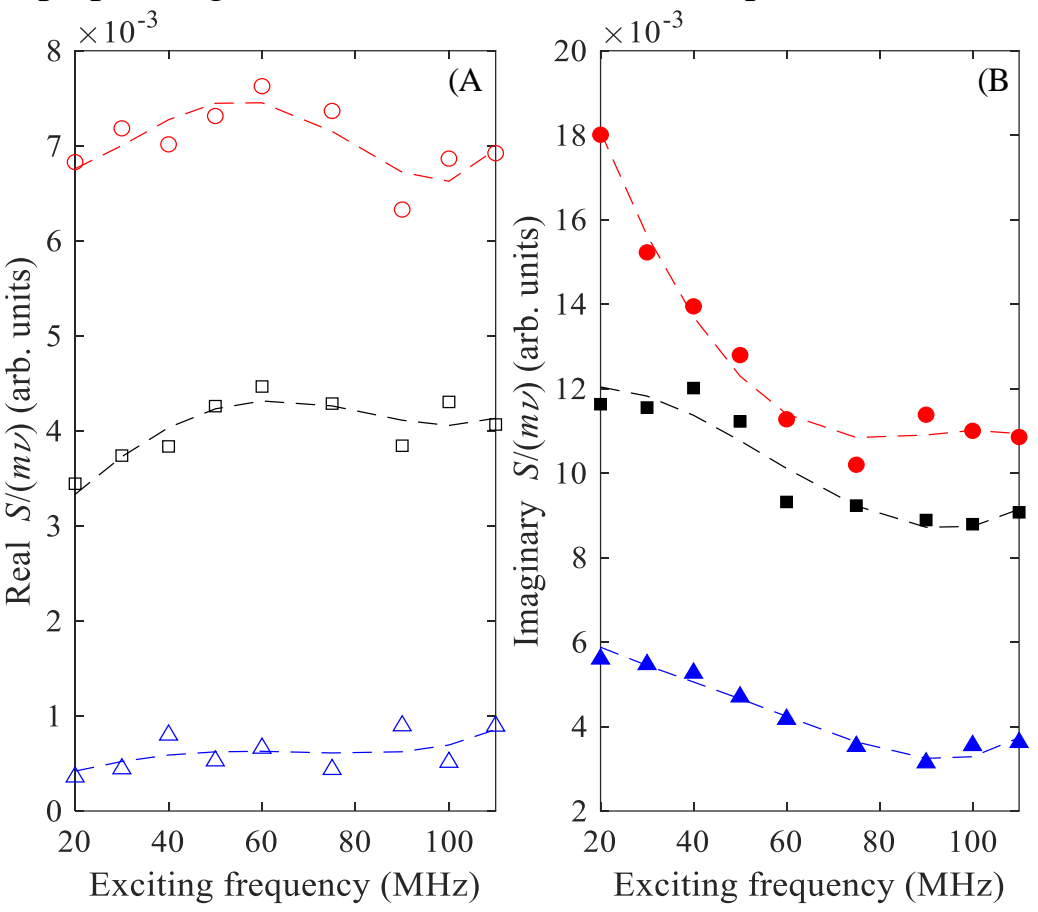

Figure 7. Real (A) and imaginary (B) components of the sensor signal divided by the measuring frequency corresponding to samples NP8 (black), NP12 (red) and NP23 (blue). The units of the vertical axes are $\Omega \cdot \mathrm{mm} /(\mathrm{mg} \cdot \mathrm{MHz})$. The dashed lines are a guide to the eye.

\subsection{Nanoparticles as Labels}

It is important to note that in the previous sections the particles were studied before biofunctionalization. This process is essential to capture the bioreceptor conjugated with its magnetic label at the test line. The superficial modification of this step involves activation of the carboxylic groups of the DMSA outer layer, which can imply some agglomeration of the particles. This, in turn, affects the number of particles that attach to each protein and, in consequence, the signal of the magnetic LFA through parameter $\psi$ in (2).

To study this effect in the nitrocellulose strips, we tested NP8, NP12, and NP23 after functionalization. For comparison, all the processes have also been performed on NP12 after six months of settling (named sample NP12A.)

To analyze the agglomeration of particles caused by the biofunctionalization or other reasons, we measured their size by TEM and compared it to the DLS results. Table 2 displays the hydrodynamic size before $\left(D_{D L S}^{B B}\right)$ and after $\left(D_{D L S}^{A B}\right)$ biofunctionalization with their corresponding polydispersity index PDI (this dimensionless parameter is used in DLS to describe the width of the size distribution; values smaller than 0.05 are associated with highly monodisperse standards, while values above 0.7 correspond to broad size distributions, ) and the mean TEM diameter $D_{0}$. For NP12 and NP23 the difference between $D_{0}$ and $D_{D L S}^{B B}$ is only $10-15 \%$, typical for the diffuse layer and surfactant around the particles. ${ }^{43}$ It indicates that these are stable suspensions of single particles. On the other hand, for NP8 and NP12A, the difference is substantial, evidencing aggregation. After the biofunctionalization, the size of NP8 and NP12 remains almost 
constant. Its small increase is due to the protein, whose size is around $5 \mathrm{~nm} \cdot{ }^{44}$ On the contrary, the size increase produced by the addition of neutravidin in NP23 and NP12A is much larger, indicating the post-functionalization agglomeration of the particles.

We conclude that there are three ways of agglomeration (schematized in Figure 8): (i) The smallest particles, NP8, aggregate in the process of transferring them to water by DMSA coating; (ii) The intermediate-sized particles, NP12, agglomerate only after long storage, as in NP12A; (iii) The largest particles, NP23, aggregate after neutravidin attachment. This could be caused by cross-linking effects due to the activation of the $\mathrm{NH}_{2}$ groups of the neutravidin. For the bioconjugation, we used a fixed neutravidin concentration per particle volume. Given that the surface area per unit volume is smaller in the larger particles, the amount of neutravidin per surface area is more significant, and could result in interparticle bridging through their $-\mathrm{COOH}$ groups.

Table 2. Mean particle diameter by TEM $D_{0}$ and its standard deviation $\sigma$; hydrodynamic diameter before biofunctionalization $D_{D L S}^{B B}$ and after biofunctionalization $D_{D L S}^{A B}$, and their corresponding PDI values.

\begin{tabular}{|l|l|l|l|l|l|l|}
\hline $\begin{array}{l}\text { Particle } \\
\text { Sample }\end{array}$ & $\begin{array}{l}\boldsymbol{D}_{\mathbf{0}} \\
(\mathbf{n m})\end{array}$ & $\begin{array}{l}\sigma \\
(\mathbf{n m})\end{array}$ & $\begin{array}{l}\boldsymbol{D}_{\boldsymbol{D} \boldsymbol{L} \boldsymbol{S}}^{\boldsymbol{B}} \\
(\mathbf{n m})\end{array}$ & PDI & $\begin{array}{l}\boldsymbol{D}_{\boldsymbol{D} \boldsymbol{A} \boldsymbol{S}}^{\boldsymbol{B}} \\
(\mathbf{n m})\end{array}$ & PDI \\
\hline NP8 & 7.6 & 0.15 & 94 & 0.36 & 106 & 0.20 \\
\hline NP12 & \multirow{2}{*}{11.6} & 0.08 & 22 & 0.20 & 34 & 0.26 \\
\cline { 1 - 5 } NP12A & & 134 & 0.39 & 260 & 0.28 \\
\hline NP23 & 22.6 & 0.11 & 44 & 0.35 & 100 & 0.33 \\
\hline
\end{tabular}

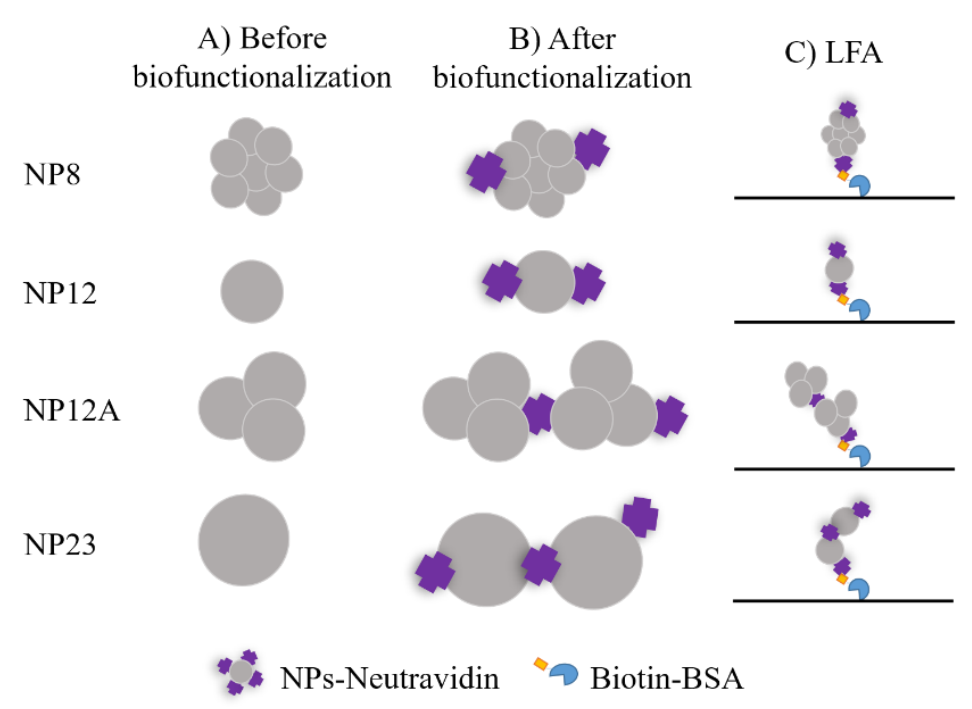

Figure 8. Schematic representation of the MNPs agglomeration (A) before and (B) after the functionalization with neutravidin, and (C) immobilized on the strip.

Figure 9 shows the magnetic sensor signal of the LFA run with different nanotags. In it, we observe that, despite NP12 having the largest magnetic susceptibility, the performance in the LFA is better for NP8 and NP23. Finally, NP12A has the best signal at all frequencies. The explanation may be agglomeration effects, as mentioned above. 
with few particles in the case of NP12A and NP23 and many more for NP8. As the number of anchoring biotin units immobilized in the test line is the same for the four cases, the signal would necessarily be smaller for NP12 than NP8 and NP23.

On the other hand, for the same cluster size, the mass of NP23 is larger, and this balances the smaller signal per unit mass. As a result, the signals recorded for NP23 are as large as those for NP8. Finally, NP12A encompasses both advantages, the highest magnetic susceptibility plus agglomeration, yielding the best signal at all frequencies.

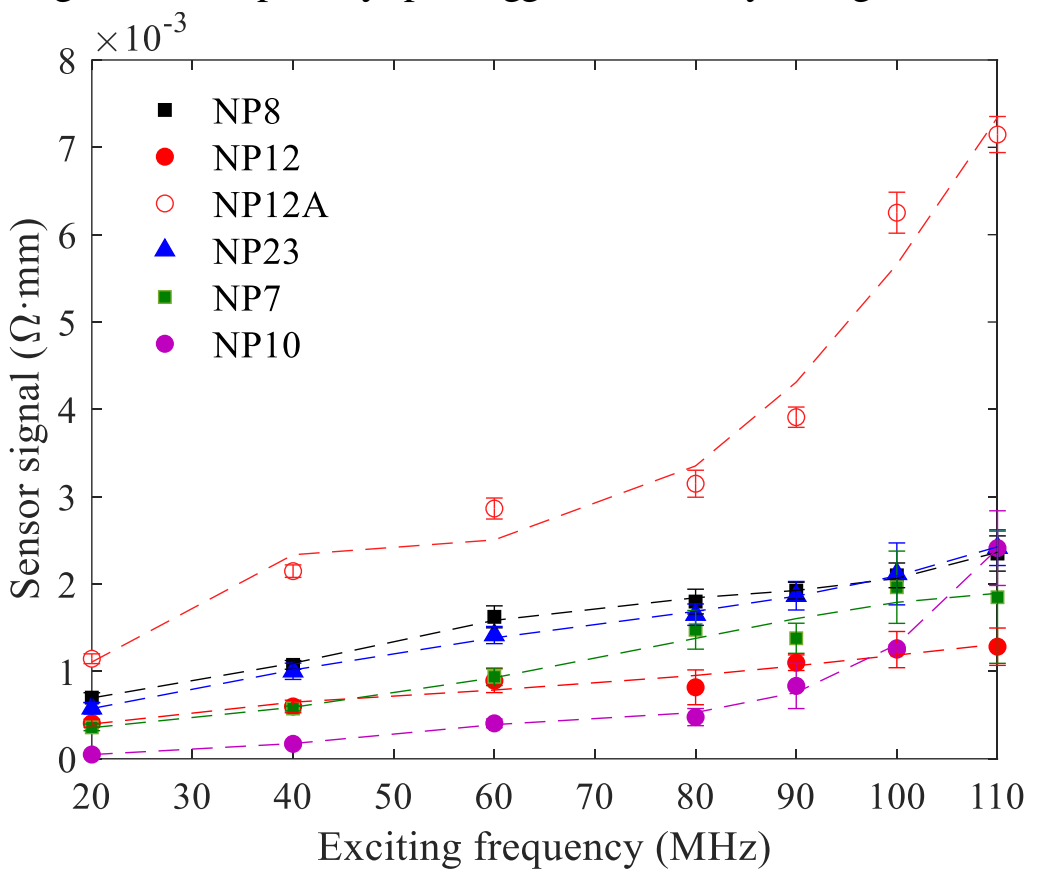

Figure 9. Magnetic signal obtained in the sensor at different frequencies for LFA with the three series of particles. The error bars show the standard deviation. The dashed lines serve as a guide to the eye.

Thus, particle clustering is crucial to enhance the performance of the magnetic LFA and can have an even greater influence than MNP permeability and saturation magnetization. As long as the aggregates are small enough to flow through the membrane pores, some agglomeration is beneficial for the detection of the MNPs. ${ }^{18}$ As a consequence, further development should include the controlled agglomeration or encapsulation of $12 \mathrm{~nm}$ sized nanoparticles, which will optimize simultaneously $\psi$ and $\chi$ in equation (2).

To evaluate the practical implications of these results, we have also performed neutravidin-biotin LFA with nanoparticles that had been used in other biological assays (Figure 9 shows these results.) Specifically, NP7 was used for histamine levels determination in red wine by LFA, achieving results that agreed with the much more complex high-performance liquid chromatography. ${ }^{20}$ Such sample consisted of $7 \mathrm{~nm}$ iron oxide MNPs forming clusters of $90 \mathrm{~nm}$. We can see in Figure 9 that MNPs with a size of $12 \mathrm{~nm}$ and clustering of $200 \mathrm{~nm}$, like NP12A, promise to improve histamine detection by LFA. We also show the results for NP10, a commercial sample of $10 \mathrm{~nm}$-sized iron oxide MNPs, which formed agglomerates of $75 \mathrm{~nm}$ after bioconjugation. Such particles were used for prostate-specific antigen quantification (the neutravidin-MNP complexes were conjugated to a biotinylated antibody) in the range of clinical interest and the measurement achieving sensitivity limits of detection comparable to ELISA and a 
resolution of $50 \mathrm{pg}$ of PSA. ${ }^{27}$ For NP12A, the resolution in MNPs detection is the same as that of NP10, $R=0.58 \mu \mathrm{g}$ (Figure 3); but, taking into account the cluster size of 260 $\mathrm{nm}$ (Table 2), a density equal to the $64 \%$ that of magnetite (accounting for a dense packed agglomeration of spheres), and assuming a binding of one PSA molecule per cluster, the estimated resolution for PSA measurements could be improved to $1 \mathrm{pg}$.

\section{Conclusions}

Lateral flow assays for the detection of clinical and environmental analytes have significant advantages compared to more sophisticated techniques in terms of speed, cost, and portability. To further extend their use, we aimed to improve their sensitivity with magnetic materials and detection. For this study, we incorporated magnetic iron oxide particles as tags that can be quantified by an inductive sensor. We analyzed the influe nce of particle size and agglomeration on the LFA magnetic readings.

Superparamagnetism of the particles is crucial for high initial permeability at the working frequencies. However, larger particles increase both the magnetic permeability and the saturation magnetization. As a consequence, the optimal size for LFA is the critical threshold for superparamagnetism. For iron oxide, this is about $12 \mathrm{~nm}$. Once this is optimized, the agglomeration of the particles before running the LFA has an enormous positive influence on the inductive measurement because it increases the magnetic moment captured at the test line. The larger the magnetic moment per biomolecule (in this case, per biotin), the larger the sensitivity of the assay. Nevertheless, this cannot be achieved by increasing the particle size, because that would eliminate the superparamagnetic behavior. The way to achieve this is to agglomerate particles with the adequate critical superparamagnetic size. Then, we conclude that $12 \mathrm{~nm}$ particles agglomerated in clusters of 200 to $300 \mathrm{~nm}$ give the best results in inductively-read magnetic LFA.

\section{Declaration of Competing Interest}

The authors declare that they have no conflict of interest.

\section{Acknowledgments}

This research was funded by the Spanish Ministry of Economy and Competitiveness under grants MAT2017-88148-R and MAT2017-84959-C2-1-R; the Council of GijónIUTA under grant SV-19-GIJÓN-1-25; and the Principality of Asturias under project IDI/2018/000185. A. Moyano and M. Salvador thanks University of Oviedo and the Principality of Asturias for their pre-doctoral grants.

\section{References}

1. P. Brangel, A. Sobarzo, C. Parolo, B. S. Miller, P. D. Howes, S. Gelkop, J. J. Lutwama, J. M. Dye, R. A. McKendry, L. Lobel and M. M. Stevens, ACS Nano, 2018, 12, 63-73.

2. R. Ranjan, S. D. Narnaware, K. Nath, R. K. Sawal and N. V. Patil, Tropical Animal Health and Production, 2018, 50, 907-910.

3. W. Ren, S. I. Mohammed, S. Wereley and J. Irudayaraj, Analytical Chemistry, 2019, 91, 2876 2884. 
4. D. Zhang, L. Huang, B. Liu, H. Ni, L. Sun, E. Su, H. Chen, Z. Gu and X. Zhao, Biosensors and Bioelectronics, 2018, 106, 204-211.

5. M. Magiati, V. M. Myridaki, T. K. Christopoulos and D. P. Kalogianni, Food Chemistry, 2019, 274, 803-807.

6. S. C. Razo, V. G. Panferov, I. V. Safenkova, Y. A. Varitsev, A. V. Zherdev and B. B. Dzantiev, Analytica Chimica Acta, 2018, 1007, 50-60.

7. D. Wang, J. Zhu, Z. Zhang, Q. Zhang, W. Zhang, L. Yu, J. Jiang, X Chen, X. Wang and P. Li, Toxins, 2019, 11, 56.

8. M. Khater, A. de la Escosura-Muñiz and A. Merkoçi, Biosensors and Bioelectronics, 2017, 93, 72-86.

9. J. Singh, S. Sharma and S. Nara, Food Chemistry, 2015, 170, 470-483.

10. S. E. F. Melanson, M. J. Tanasijevic and P. Jarolim, Circulation, 2007, 116, e501-e504.

11. Y. He, S. Zhang, X Zhang, M. Baloda, A. S. Gurung, H. Xu, X Zhang and G. Liu, Biosensors and Bioelectronics, 2011, 26, 2018-2024.

12. D. H. Choi, S. K. Lee, Y. K. Oh, B. W. Bae, S. D. Lee, S. Kim, Y.-B. Shin and M.-G. Kim, Biosensors and Bioelectronics, 2010, 25, 1999-2002.

13. N. M. Rodriguez, W. S. Wong, L. Liu, R. Dewar and C. M. Klapperich, Lab on a Chip, 2016, 16, 753-763.

14. A. Beninato, V. Sinatra, G. Tosto, M. E. Castagna, S. Petralia, S. Conoci and S. Baglio, IEEE Transactions on Instrumentation and Measurement, 2017, 66, 348-359.

15. M. O. Rodríguez, L. B. Covián, A. C. García and M. C. Blanco-López, Talanta, 2016, 148, 272-278.

16. Katarzyna M. Koczula and A. Gallotta, Essays In Biochemistry, 2016, 60, 111-120.

17. M. A. Mansfield, in Drugs of Abuse: Body Fluid Testing, eds. R. C. Wong and H. Y. Tse, Humana Press, Totowa, NJ, 2005, DOI: 10.1007/978-1-59259-951-6_4, pp. 71-85.

18. Y. Wang, H. Xu, M. Wei, H. Gu, Q. Xu and W. Zhu, Materials Science and Engineering: $C$, 2009, 29, 714-718.

19. G. Ruiz-Vega, M. Kitsara, M. A. Pellitero, E. Baldrich and F. J. del Campo, ChemElectroChem, 2017, 4, 880-889.

20. A. Moyano, M. Salvador, J. C. Martínez-García, V. Socoliuc, L. Vékás, D. Peddis, M. A. Alvarez, M. Fernández, M. Rivas and M. C. Blanco-López, Analytical and Bioanalytical Chemistry, 2019, 411, 6615-6624.

21. C. R. Tamanaha, S. P. Mulvaney, J. C. Rife and L. J. Whitman, Biosensors and Bioelectronics, 2008, 24, 1-13.

22. V. G. Panferov, I. V. Safenkova, A. V. Zherdev and B. B. Dzantiev, Talanta, 2017, 164, 6976.

23. K. Aguilar-Arteaga, J. A. Rodriguez and E. Barrado, Analytica Chimica Acta, 2010, 674, 157165.

24. X. Zhao, Y. Shi, Y. Cai and S. Mou, Environmental Science \& Technology, 2008, 42, 1201 1206.

25. D. Quesada-González and A. Merkoçi, Biosensors and Bioelectronics, 2015, 73, 47-63.

26. D. Lago-Cachón, M. Rivas, J. C. Martínez-García and J. A. García, Nanotechnology, 2013, 24, 245501.

27. D. Lago-Cachón, M. Oliveira-Rodríguez, M. Rivas, M. C. Blanco-López, J. C. Martínez-García, A. Moyano, M. Salvador and J. A. García, IEEE Magnetics Letters, 2017, 8, 1-5.

28. L. M. Bronstein, X Huang, J. Retrum, A. Schmucker, M. Pink, B. D. Stein and B. Dragnea, Chemistry of Materials, 2007, 19, 3624-3632.

29. G. Salas, C. Casado, F. J. Teran, R. Miranda, C. J. Serna and M. P. Morales, Journal of Materials Chemistry, 2012, 22, 21065-21075.

30. R. Mejías, L. Gutiérrez, G. Salas, S. Pérez-Yagüe, T. M. Zotes, F. J. Lázaro, M. P. Morales and D. F. Barber, Journal of Controlled Release, 2013, 171, 225-233.

31. T. T. Le, P. Chang, D. J. Benton, J. W. McCauley, M. lqbal and A. E. G. Cass, Analytical Chemistry, 2017, 89, 6781-6786.

32. Z. Fang, W. Wu, X Lu and L. Zeng, Biosensors and Bioelectronics, 2014, 56, 192-197.

33. K. V. Serebrennikova, J. V. Samsonova and A. P. Osipov, Moscow University Chemistry Bulletin, 2018, 73, 131-134.

34. A. Ruiz, G. Salas, M. Calero, Y. Hernández, A. Villanueva, F. Herranz, S. Veintemillas Verdaguer, E. Martínez, D. F. Barber and M. P. Morales, Acta Biomaterialia, 2013, 9, 6421-6430.

35. C. P. Bean and J. D. Livingston, Journal of Applied Physics, 1959, 30, S120-S129. 
36. R. Egli, Geophysical Journal International, 2009, 177, 395-420.

37. H. Zhang, D. Zeng and Z. Liu, Journal of Magnetism and Magnetic Materials, 2010, 322 , 2375-2380.

38. R. M. Cornell and U. Schwertmann, The iron oxides: structure, properties, reactions, occurrences and uses, John Wiley \& Sons, 2003.

39. D. Peddis, M. V. Mansilla, S. Mørup, C. Cannas, A. Musinu, G. Piccaluga, F. D'Orazio, F. Lucari and D. Fiorani, The Journal of Physical Chemistry B, 2008, 112, 8507-8513.

40. M. F. Hansen and S. Mørup, Journal of Magnetism and Magnetic Materials, 1999, 203, 214216.

41. M. Knobel, W. C. Nunes, L. M. Socolovsky, E. De Biasi, J. M. Vargas and J. C. Denardin, Journal of nanoscience and nanotechnology, 2008, 8, 2836-2857.

42. J.-H. Lee, J.-t. Jang, J.-s. Choi, S. H. Moon, S.-h. Noh, J.-w. Kim, J.-G. Kim, I.-S. Kim, K. I. Park and J. Cheon, Nature Nanotechnology, 2011, 6, 418-422.

43. R. D. Boyd, S. K. Pichaimuthu and A. Cuenat, Colloids and Surfaces A: Physicochemical and Engineering Aspects, 2011, 387, 35-42.

44. C. Rosano, P. Arosio and M. Bolognesi, Biomolecular Engineering, 1999, 16, 5-12. 